\title{
Method of evaluating the level of confidence based on metrological risks for determining the coverage factor in the concept of uncertainty
}

\author{
Oleksandr Vasilevskyi, Volodymyr Didych, Anna \\ Kravchenko, Maksym Yakovlev, Iryna Andrikevych, et \\ al.
}

Oleksandr Vasilevskyi, Volodymyr Didych, Anna Kravchenko, Maksym Yakovlev, Iryna Andrikevych, Dmytro Kompanets, Yevhen Danylyuk, Waldemar Wójcik, Askhat Nurmakhambetov, "Method of evaluating the level of confidence based on metrological risks for determining the coverage factor in the concept of uncertainty ," Proc. SPIE 10808, Photonics Applications in Astronomy, Communications, Industry, and High-Energy Physics Experiments 2018, 108082C (1 October 2018); doi: 10.1117/12.2501576

SPIE Event: Photonics Applications in Astronomy, Communications, Industry, and High-Energy Physics Experiments 2018, 2018, Wilga, Poland 


\title{
Method of evaluating the level of confidence based on metrological risks for determining the coverage factor in the concept of uncertainty
}

\author{
Oleksandr Vasilevskyi*a ${ }^{\text {a }}$ Volodymyr Didych $^{\mathrm{b}}$, Anna Kravchenko ${ }^{\mathrm{c}}$, Maksym Yakovlev ${ }^{\mathrm{d}}$, \\ Iryna Andrikevych ${ }^{\mathrm{b}}$, Dmytro Kompanets ${ }^{\mathrm{a}}$, Yevhen Danylyuk ${ }^{\mathrm{a}}$, Waldemar Wójcik ${ }^{\mathrm{e}}$, \\ Askhat Nurmakhambetov ${ }^{\mathrm{f}}$ \\ ${ }^{a}$ Vinnytsia National Technical University, 95 Khmelnytske, Vinnytsia, 21021, Ukraine; ${ }^{b}$ Vinnytsia \\ National Medical University named after M. I. Pirogov, 56 Pirogova, Vinnitsa, 21018, Ukraine; \\ 'National University of Life and Environmental Sciences of Ukraine, 15 Heroyiv Oborony, Kyiv, \\ 03041, Ukraine; ${ }^{\mathrm{d}}$ Hetman Petro Sahaidachny National Army Academy, 32 Heroiv Maidanu, Lviv, \\ 79000, Ukraina; ${ }^{2}$ Lublin University of Technology, 38A Nadbystrzycka Str., Lublin, 20-618, Poland; \\ ${ }^{\mathrm{f}}$ Kazakh Academy of Transport \& Communication, 97 Shevchenko Str., Almaty, 050012, \\ Kazakhstan
}

\begin{abstract}
A method for estimating the level of confidence for determining the coverage factor based on metrological risks is proposed using the example of using information on tolerances and uncertainty of measuring the activity of ions, which allows to establish a reasonable interval around the measurement result, within which most of the values that can be justified are assigned to the measured value.
\end{abstract}

Keywords: measurements, uncertainty, level of confidence, metrological risks, activity of ions, expanded uncertainty

\section{INTRODUCTION}

International standards JCGM 100:2008 (GUM 1995 with minor corrections), JCGM 102:2011, JCGM 104:2009, ISO/IEC Guide 98-1:2009 "Uncertainty of measurement - Part 1: Introduction to the expression of uncertainty in measurement", support the use of combined uncertainty uc (y) as a parameter for quantifying the uncertainty of the measurement result ${ }^{1-4}$. Although the combined uncertainty uc $(\mathrm{u})$ can be widely used to express the uncertainty of the measurement result, however, in some cases: in trade, industry and regulatory acts, and when it comes to health and safety, it is advisable to further specify an interval measure of uncertainty that determines the interval for the measurement result. An additional measure of uncertainty, which corresponds to interval estimation of uncertainty, is called expanded uncertainty. Therefore, the task of evaluating the trust level for calculating the expanded measurement uncertainty with the use of a particular measurement tool is relevant.

The purpose of the article is to develop a mathematical apparatus for estimating the confidence level in calculating the expanded uncertainty of measuring the activity of ions taking into account the manufacturer's (developer's) and consumer metrological risks, which will enable to establish the interval around the measurement result of the ion activity, within which the majority of the distribution of values which can be attributed to the measured value.

\section{MAIN MATERIALS OF THE RESEARCH}

In literary sources ${ }^{1-13}$, only a partial consideration is given to ways of establishing a trust level for calculating the expanded uncertainty of measurement. The mathematical apparatus that would allow reasonable confidence level set in the concept of measurement uncertainty is not described. It is therefore advisable to propose and describe a methodology for estimating trust level based on the metrological risks of the manufacturer and the consumer, which will allow to establish the value of the coefficient of coverage $\mathrm{k}$ for the calculation of the expanded uncertainty of measurement on an example of the use of the developed means for measuring the activity of ions.

*o.vasilevskyi@gmail.com 
The confidence level for calculating the expanded uncertainty is proposed based on the metrological risks of the manufacturer and the consumer by the formula

$$
D=1-P_{n}=1-(\alpha+\beta),
$$

where $\alpha$ is metrological risk of the manufacturer; $\beta$ is metrological risk of the consumer; $P_{n}$ is the total value of metrological risk.

The procedure for determining the confidence level is described on the basis of using the combined uncertainty of the results of reusable measurements of ion activity using a measuring instrument built on the principle of converting voltage to frequency ${ }^{14}$, which is described by such a transformation equation

$$
N_{\boldsymbol{U} / \boldsymbol{F}}=\frac{U_{\text {pow }} f_{0} \tau}{\left(U_{0}-\frac{\alpha_{t}(273.15+t)}{n_{a}} p X_{i}\right) k_{2}} .
$$

where $\mathrm{p} \mathrm{X}_{\mathrm{i}}$ is activity of ions; $\alpha_{t}$ is temperature coefficient of steepness, equal to $198.4 \cdot 10^{-3}{ }^{\circ} \mathrm{C}^{-1} ; \mathrm{t}$ is the temperature of the medium under investigation; $k_{2}$ is gain scaling factor (DA1, DA2, Fig. 1); $U_{\text {pow }}$ is value of the reference voltage of the voltage converter in the frequency $(10 \mathrm{~V}) ; \tau=\mathrm{RC}$ is the time constant of the voltage converter in a frequency that is used to set the full scale output frequency range $(\mathrm{R}=1 \mathrm{kOm}, \mathrm{C}=47 \mu \mathrm{F}) ; \mathrm{f}_{0}$ is frequency of the quartz resonator microcontroller $(20 \mathrm{MHz}) U_{0}$ is the standard potential of the reference electrode at the initial isopotential point.; $n_{a}$ is charge of the i-th ion.

The electrical functional circuit of the ion activity measuring device built on the conversion of voltage to frequency is shown in Figure 1.

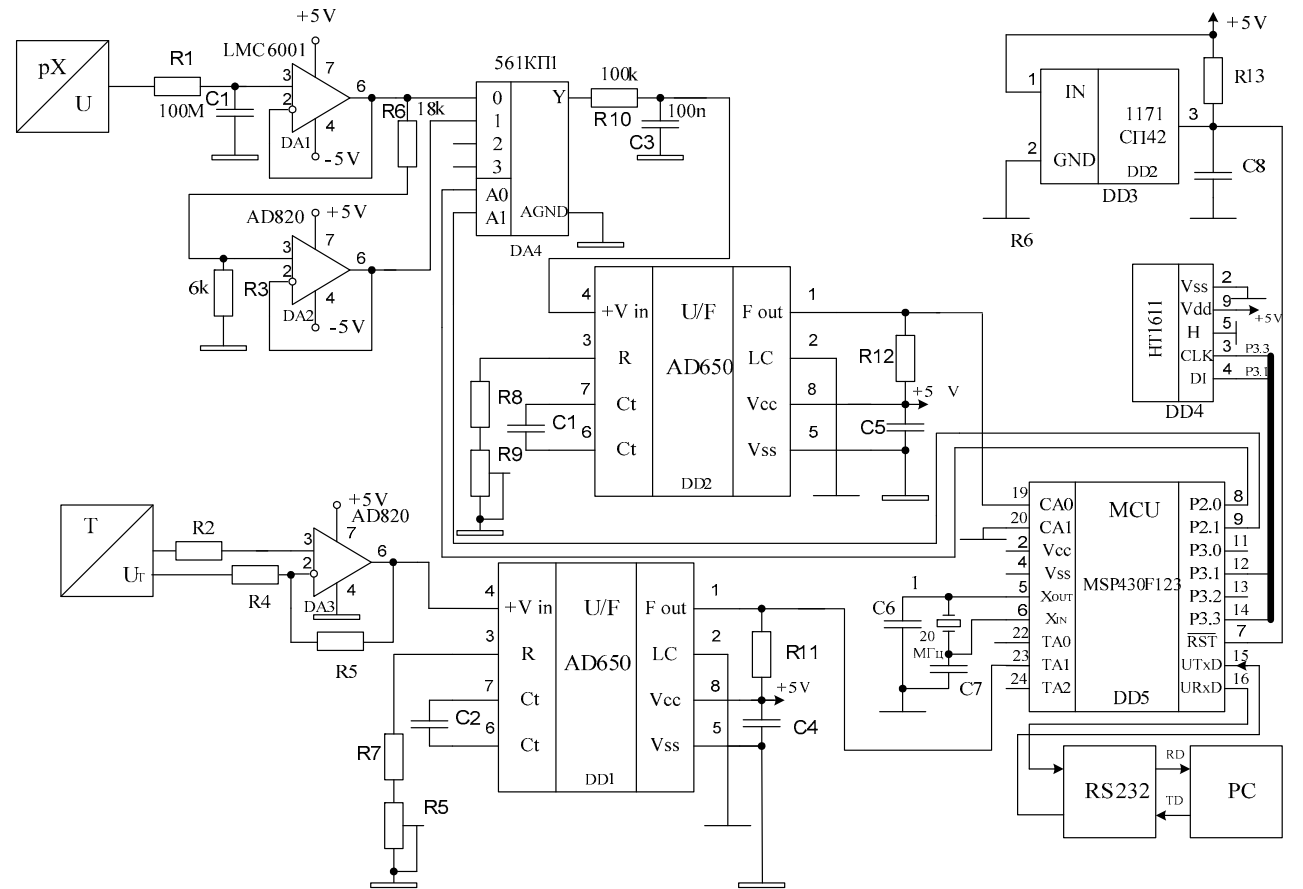

Figure 1. The electrical functional circuit of the ion activity measuring device.

The general law of uncertainty of measuring ion activity depends on many factors such as activity interfering ions limited properties of ion-selective electrodes, the presence of measurement error in temperature, zero drift, instability of power supply, etc., among which is difficult to identify dominant ${ }^{14}$. This allows us to adopt the law of distribution of the centered value of the error of measuring the activity of ions in the normal, which we describe with the expression 


$$
p(p X)=\frac{1}{u_{c}(p X) \sqrt{2 \pi}} \exp \left(-\frac{(p X-p \bar{X})^{2}}{2 u_{c}^{2}(p X)}\right)
$$

Where $p \bar{X}$ is estimation of ion activity value; $\mathrm{u}_{\mathrm{c}}(\mathrm{pX})$ is combined uncertainty of measurement ion activity.

The value of the combined uncertainty of measuring ion activity when using a measuring device built on the basis of the conversion of voltage to frequency is calculated by the formula

$$
\begin{gathered}
u_{c}^{2}(p X)=u_{A}{ }^{2}(p X)+u_{c B}^{2}, \\
u_{c B}^{2}=\sum_{i=1}^{N} c_{i}^{2} u_{i}^{2}(p X)+2 \sum_{j=1}^{N-1} \sum_{i=2}^{N} c_{i} c_{j} r(p X, t) u_{i}(p X) u_{j}(t),
\end{gathered}
$$

where $u_{A}(p X)$ is evaluation of standard uncertainty type $A ; u_{i}(p X)$ is components of the measurement uncertainty of activity of ions of type $B$ with the respective sensitivity coefficients $c_{i} ; u_{j}(t)$ is components of temperature measurement uncertainty of type $B$ with corresponding sensitivity coefficients $c_{j} ; r(p X, t)$ is the correlation coefficient between ion activity $(p X)$ and temperature $(t)^{14-17}$.

Taking into account the transformation equations (2), the combined uncertainty of type $B$ is determined by the formula

$$
\begin{gathered}
u_{c B 1}^{2}=\left(\frac{\partial N_{\boldsymbol{U} / \boldsymbol{F}}}{\partial p X_{i}}\right)^{2}\left[u_{B_{E}}^{2}+u_{B_{U / F}}^{2}\right]+\left(\frac{\partial N_{U / \boldsymbol{F}}}{\partial U_{0}}\right)^{2} u_{B \Theta_{U 0}}^{2}+\left(\frac{\partial N_{\boldsymbol{U} / \boldsymbol{F}}}{\partial t}\right)^{2} u_{B_{P}}^{2}+\left(\frac{\partial N_{\boldsymbol{U} / \boldsymbol{F}}}{\partial U_{p o w}}\right)^{2} u_{B_{\boldsymbol{U}} \boldsymbol{p o w}}^{2}, \\
u_{c B}^{2}=u_{c B 1}^{2}+2 \frac{\partial N_{U / \boldsymbol{F}}}{\partial p X_{i}} u_{c B 1} \frac{\partial N t_{\boldsymbol{U} / \boldsymbol{F}}}{\partial t} u_{c B t} \frac{\sum_{l=1}^{n_{i j}}\left(t_{i l}-\overline{t_{i}}\right)\left(p X_{j l}-\overline{p X_{j}}\right)}{\sqrt{\sum_{l=1}^{n_{i j}}\left(t_{i l}-\bar{t}_{i}\right)^{2} \sum_{l=1}^{n_{i j}}\left(p X_{j l}-\overline{p X_{j}}\right)^{2}}},
\end{gathered}
$$

where $N t_{U / F}=\frac{4 U_{\text {pow }} f_{0} \tau}{k_{1} E_{s} \alpha_{t} t}$ is the equation of transformation of a measuring channel of temperature (Figure 1) $)^{14} ; \mathrm{E}_{\mathrm{s}}=\mathrm{IR}_{0}$ is sensor supply voltage $\left(R_{0}\right.$ is sensor resistance at temperature $\left.0^{\circ} \mathrm{C}\right)$; $\mathrm{k}_{1}$ is gain scaling factor (DA3, Fig. 1); $\frac{\partial N_{U / F}}{\partial p X_{i}}=\frac{\alpha_{t}(273.15+t) U_{p o w} f_{0} \tau}{\left[U_{0}-\frac{\alpha_{t}(273.15+t)}{n_{a}} p X_{i}\right]^{2} k_{2} n_{a}}=12.98\left[\mathrm{pX}^{-1}\right]$ is sensitivity factor for ion activity at a temperature of $25^{\circ} \mathrm{C}$; $\frac{\partial N_{U / F}}{\partial U_{0}}=-\frac{U_{p o w} f_{0} \tau}{\left[U_{0}-\frac{\alpha_{t}(273.15+t)}{n_{a}} p X_{i}\right]^{2} k_{2}}=-0.22\left[\mathrm{~V}^{-1}\right]$ is coefficient of voltage sensitivity of the standard potential of the reference electrode; $\frac{\partial N_{U / F}}{\partial t}=\frac{\alpha_{t} p X_{i} U_{p o w} f_{0} \tau}{\left[U_{0}-\frac{\alpha_{t}(273,15+t)}{n_{a}} p X_{i}\right]^{2} k_{2} n_{a}}=0.3\left[{ }^{\circ} \mathrm{C}^{-1}\right]$ is coefficient of sensitivity at an additional measured temperature; $\frac{\partial N_{U / F}}{\partial U_{\text {pow }}}=\frac{f_{0} \tau}{\left(U_{0}-\frac{\alpha_{t}(273,15+t)}{n_{a}} p X_{i}\right) k_{2}}=-9.08\left[\mathrm{~V}^{-1}\right]$ is coefficient of voltage sensitivity of the source of stable power supply of the voltage converter to the frequency; $n_{i j}$ is the number of pairwise measurements of 
the temperature and ion activity; $u_{B_{E}}=\gamma \frac{p X_{\max }}{100 \% \sqrt{3}}=1.21 \cdot 10^{-3}[\mathrm{pX}]$ is uncertainty of type $\mathrm{B}$, which is due to the presence of a limited class of accuracy $(\gamma=0.7 \%)$ of the primary electrode; $u_{B \Theta_{U 0}}=\frac{\Theta_{U 0}}{\sqrt{3}}=0.95[\mathrm{mV}]$ is uncertainty of type $\mathrm{B}$, which is caused by the instability $\left(\Theta_{U 0}=1.65 \mathrm{mV}\right)$ of the standard potential of the reference electrode; $u_{B p}=\frac{\Delta t \text { Ubias }_{\max }}{U_{\text {Pout }} \sqrt{3}} \approx 2.89 \cdot 10^{-3}\left[{ }^{\circ} \mathrm{C}\right]$ is uncertainty of type $\mathrm{B}$, which is caused by the existence of a bias voltage $\left(\right.$ Ubias $\left._{\text {max }} / U_{\text {Pout }}=0.2 \cdot 10^{-3}\right)$ of the operational amplifier when the temperature deviates $\left(\Delta t=5^{\circ} \mathrm{C}\right)$ from the nominal value; $u_{B_{U_{P O W}}}=\frac{\Theta_{U_{P}}}{\sqrt{3}} \approx 1.16[\mathrm{mV}]$ is uncertainty of type $\mathrm{B}$, which is caused by instability of the power supply source of the reference voltage $\left(\Theta_{U_{P}}=2 \mathrm{mV}\right) ; u_{B_{\Pi H}}=\frac{\delta_{\text {nonl }} p X_{\max }}{100 \% \sqrt{3}}=3.5 \cdot 10^{-6}[\mathrm{pX}]$ is uncertainty of type B, which is caused by the nonlinearity $\left(\delta_{\text {nonl }}\right)$ of the voltage converter in a frequency $(\mathrm{AD} 650)^{14-18}$.

The values of the combined uncertainty are calculated in ${ }^{14}$ and its maximum value for measuring the activity of phosphate ions is $17.21 \cdot 10^{-3} \mathrm{pX}$ in the range of measurements from 6 to $0.3 \mathrm{pX}$.

A compatible two-dimensional confidence level density when measuring the activity of ions, taking into account the allowable deviation of the measurement error $\varepsilon$, which is established by the consumer, is described by the expression

$$
p(p X, \varepsilon)=\frac{1}{2 \pi \cdot u_{c}(p X) \cdot u_{\varepsilon}} \exp \left(-\frac{(p X-p \bar{X})^{2}}{2 u_{c}^{2}(p X)}-\frac{(\Delta \varepsilon)^{2}}{2 u_{\varepsilon}^{2}}\right) .
$$

In most practical cases, as noted in $^{8,18}$, the admissible deviation $\varepsilon$ of the parameter which is controlled by the user, depending on the combined uncertainty of the measurement results $u_{c}(y)$, is determined by the formula

$$
\varepsilon[\%]=\frac{6 u_{c}(y)}{d} 100=\frac{6 u_{c}(y)}{X_{\max }-X_{\min }} 100,
$$

where $d$ is width of the tolerance field determined by the values of the upper $X_{\max }$ and lower $X_{\min }$ range of measurement of the physical quantity.

Substituting the values of the maximum combined uncertainty of measuring the ion activity $\mathrm{u}_{\mathrm{c}}(\mathrm{pX})=17.21 \cdot 10^{-3} \mathrm{pX}$ and the value of the upper $\mathrm{X}_{\max }=6 \mathrm{pX}$ and the lower $\mathrm{X}_{\min }=0.3 \mathrm{pX}$ of the measurement limit in equation (9), we obtain the values of the permissible error set by the consumer, which is $\varepsilon=1.81 \%$.

The uncertainty $u_{\varepsilon}$ of the tolerance field of the controlled parameter at which the result can be considered reliable in practice is recommended to be equal to $6 \mathrm{u}_{\mathrm{c}}(\mathrm{y})^{18}$. Thus accept $\mathrm{u}_{\varepsilon}=6 \cdot \mathrm{u}_{\mathrm{c}}(\mathrm{y})=6 \cdot 17.21 \cdot 10^{-3}=103.26 \cdot 10^{-3} \mathrm{pX}$.

Taking into account the expression (8), the metrological risk of the manufacturer $\alpha$ is estimated by the formula

$$
\alpha=\int_{X_{\min }}^{X_{\max }}\left[\int_{-\infty}^{X_{\min }^{+}+\Delta p X} \frac{\exp \left(-\frac{(\Delta p X)^{2}}{2 u_{c}^{2}(p X)}-\frac{(\Delta \varepsilon)^{2}}{2 u_{\varepsilon}^{2}}\right)}{2 \pi \cdot u_{c}(p X) \cdot u_{\varepsilon}} d \Delta \varepsilon+\int_{X_{\max }-\Delta p X}^{\infty} \frac{\exp \left(-\frac{(\Delta p X)^{2}}{2 u_{c}^{2}(p X)}-\frac{(\Delta \varepsilon)^{2}}{2 u_{\varepsilon}^{2}}\right)}{2 \pi \cdot u_{c}(p X) \cdot u_{\varepsilon}} d \Delta \varepsilon\right] d \Delta p X
$$

the metrological risk of the consumer $\beta$ is estimated by the formula

$$
\beta=\int_{-\infty}^{X_{\min }} X_{X_{\min }+\Delta p X} \frac{\Delta p X}{\exp \left(-\frac{(\Delta p X)^{2}}{2 u_{c}^{2}(p X)}-\frac{(\Delta \varepsilon)^{2}}{2 u_{\varepsilon}^{2}}\right)} d \Delta p X d \Delta \varepsilon+\int_{X_{\max }}^{\infty} \int_{X_{\min }+\Delta p X}^{\left.X_{\max }-\Delta p X\right) \cdot u_{\varepsilon}} \frac{\exp \left(-\frac{(\Delta p X)^{2}}{2 u_{c}^{2}(p X)}-\frac{(\Delta \varepsilon)^{2}}{2 u_{\varepsilon}^{2}}\right)}{2 \pi \cdot u_{c}(p X) \cdot u_{\varepsilon}} d \Delta p X d \Delta \varepsilon .
$$


The admission field is the value of the measured value $\Delta \mathrm{pX}$, in this case, this is the activity of the $\mathrm{pX}$ ions, we define by the formula

$$
\Delta p X=\frac{p \bar{X}}{100} \delta_{\max }
$$

where $p \bar{X}$ is estimation of activity of ions on the upper measurement range; $\delta_{\max }$ is the maximum relative measurement error (for the measuring channel of ion activity this value is $0.7 \%$ in the range of measurements from 6 to $0.3 \mathrm{pX}$ ).

Consequently, to calculate the metrological risks of the manufacturer and the consumer, the field of admission $\Delta \mathrm{pX}$ we will calculate by the formula (12), which when adding the corresponding numerical values is equal to $2.1 \cdot 10^{-3} \mathrm{pX}$.

Substituting calculated tolerances in the formula for estimating metrological risks manufacturer (10) and consumer (11) and solving them using the Maple 12 math package we obtain the following numerical values: $\alpha=0.24 \cdot 10^{-67}(\alpha \approx 0)$, $\beta=0.00196$. The total value of the metrological risk is $P_{n}=0.00196$, and the confidence level for calculating the expanded uncertainty of the measurement, according to formula (1) will be equal to $D=1-P_{n}=1-0.00196=0.998$.

Characteristics of the change of metrological risks of the manufacturer and the consumer depending on the parameter $\mu=u_{\varepsilon} / u_{c}(p X)$, which establishes the relationship between the permissible uncertainty of $u_{\varepsilon}$, which is specified by the consumer (normative documents) and combined uncertainty of $u_{c}(\mathrm{pX})$, which is set by the manufacturer (developer) of measuring device for $\mathrm{u}_{\varepsilon}<\mathrm{u}_{\mathrm{c}}(\mathrm{pX}), \mathrm{u}_{\varepsilon} \approx \mathrm{u}_{\mathrm{c}}(\mathrm{pX}), \mathrm{u}_{\varepsilon}>\mathrm{u}_{\mathrm{c}}(\mathrm{pX})$ are shown in Figure 2.

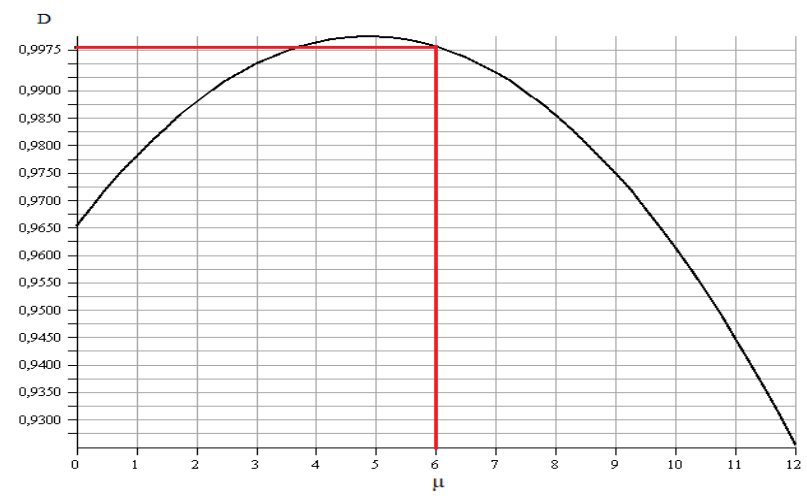

Figure 2. Characteristic of the change of metrological risk depending on the parameter $\mu$.

Thus, based on the constructed characteristic of the change in reliability (Figure 2), which is obtained by calculating the metrological risks of the consumer and the manufacturer, it is calculated, within which the majority of the distribution of values that can be attributed to the measured value is likely to be located in depending on the value of the accepted tolerance for the monitored parameter.

As can be seen from Figure 2, with a tolerance of 6 combined uncertainties, the confidence level is $99.8 \%$. Given the level of confidence based on metrological risks, the expanded uncertainty can be calculated from the formula $U=k_{p} \cdot u_{c}(y)=k_{99,8} \cdot 17,21 \cdot 10^{-3}$, where $\mathrm{k}_{\mathrm{p}}$ is coverage ratio for the established confidence level $(\mathrm{p}=\mathrm{D})$, which is taken from the Student's table.

Thus, the coverage factor $k_{p}$ with the number of degrees of freedom 30 and the confidence probability of $99.8 \%$ is 3.385 , and with the number of degrees of freedom 10 and the probability $99.8 \%-4.14$. Accordingly, substituting these values of the coverage factor in equation (13), the expanded uncertainty will be is $U_{99,8}=71,25 \cdot 10^{-3} \mathrm{pX}$ for the number of degrees of freedom 10 and $\mathrm{U}_{99,8}=58,26 \cdot 10^{-3} \mathrm{pX}$ for the number of degrees of freedom 30 .

\section{CONCLUSIONS}

The work describes the characteristics of the change in the metrological risks of the manufacturer and the consumer on the basis of which a general characteristic of the change in the metrological risk that occurs during the measurement is obtained the activity of ions on the basis of which it is possible to determine graphically the confidence level within which most of the distribution of values obtained by measuring the activity of ions is probably located. 
The obtained monography allows one to determine with a high probability the confidence level at a given tolerance for the controlled parameter to determine the coverage factor in calculating the extended uncertainty of ion activity measurement.

The described approach for determining confidence probability on the basis of metrological risks can be applied for any types of measurements provided a separate calculation of the metrological risks of the manufacturer of the measuring instrument and the consumer (risks of the first and second kind).

\section{REFERENCES}

[1] BIPM, IEC, IFCC, ISO, IUPAC, IUPAP and OIML, "Evaluation of measurement data - Guide to the expression of uncertainty in measurement," Joint Committee for Guides in Metrology - JCGM 100:2008 (2008).

[2] BIPM, IEC, IFCC, ISO, IUPAC, IUPAP and OIML, "Evaluation of Measurement Data - Supplement 2 to the Guide to the Expre ssion of Uncertainty in Measurement - Extension to any number of output quantities," Joint Committee for Guides in Metrology - JCGM 102:2011 (2011).

[3] BIPM, IEC, IFCC, ISO, IUPAC, IUPAP and OIML, "Evaluation of measurement data - An introduction to the Guide to the expression of uncertainty in measurement and related documents," Joint Committee for Guides in Metrology - JCGM 104:2009 (2009).

[4] ISO/IEC Guide 98-1:2009, "Uncertainty of measurement - Part 1: Introduction to the expression of uncertainty in measurement," (2009).

[5] Vasilevskyi, O. M., "Calibration method to assess the accuracy of measurement devices using the theory of uncertainty," International Journal of Metrology and Quality Engineering 5(4), 403 (2014).

[6] IEC GUIDE 115-2007, "Application of uncertainty of measurement to conformity assessment activities in the electrotechnical sector," (2007).

[7] ISO/IEC 17025:2005, "General requirements for the competence of testing and calibration laboratories," (2005).

[8] Beckert, S. F. and Paim, W. S., "Critical analysis of the acceptance criteria used in measurement systems evaluation," International Journal of Metrology and Quality Engineering 8, 23 (2017).

[9] Duk, M., Kociubiński, A., Bieniek, T. and Janus, P., "3-D modeling of anisotropic wet etching of silicon," Przegląd Elektrotechniczny 86, 281-283 (2010).

[10] Gotra, Z., Golyaka, R., Pavlov, S. and Kulenko, S., "High resolution differential thermometer," Technology and Design in Electronic Apparatuses 6, 19-23 (2009).

[11]Lytvyn, V. and Rishnyak, I., "Modeling and evaluation of project risks in multi-project environment," Informatyka, Automatyka, Pomiary w Gospodarce i Ochronie Środowiska - IAPGOŚ 2, 34-36 (2014).

[12] Osadchuk, A., Osadchuk, I., Smolarz, A. and Kussambayeva, N., "Pressure transducer of the on the basis of reactive properties of transistor structure with negative resistance," Proc. SPIE 9816, (2015).

[13] Semenov, A. O., Osadchuk, A. V., Osadchuk, I. A., Koval, K. O. and Prytula, M. O., "The chaos oscillator with inertial non-linearity based on a transistor structure with negative resistance," Proceedings of the International Conference Micro/Nanotechnologies and Electron Devices - EDM, (2016).

[14] Vasilevskyi, O. M. and Didych, V. M., [Elements of the theory of constructing potentiometric means of measuring control of ion activity with an increased probability], VNTU, Vinnytsa (2013).

[15] Azarov, O. D., Krupelnitskyi, L. V. and Komada, P., "AD systems for processing of low frequency signals based on self calibrate ADC and DAC with weight redundancy," Przeglad Elektrotechniczny 93(5), 125-128 (2017).

[16] Azarov, O., Chernyak, O., Komada, P., Kozhambardiyeva, M. and Kalizhanova, A., "High-speed counters in Fibonacci numerical system," Proc. SPIE 10445, (2017).

[17] Vasilevskyi, O. M., Kulakov, P. I., Dudatiev, I. A., Didych V. M., Kotyra, A., Suleimenov B., Assembay A. and Kozbekova, A., "Vibration diagnostic system for evaluation of state interconnected electrical motors mechanical parameters," Proc. SPIE 10445, (2017).

[18] Wheeler, D. J., “An Honest Gauge R\&R Study,” Proc. ASQ/ASA Fall Technical Conference 189 (2009). 